\title{
A Porous Covalent Organic Framework with Voided Square Grid Topology for Atmospheric Water Harvesting
}

Ha L. Nguyen, Nikita Hanikel, Steven J. Lyle, Chenhui Zhu, Davide M. Proserpio, and Omar M. Yaghi

J. Am. Chem. Soc., Just Accepted Manuscript • DOI: 10.1021/jacs.9b13094 • Publication Date (Web): 16 Jan 2020

Downloaded from pubs.acs.org on January 16, 2020

\section{Just Accepted}

"Just Accepted" manuscripts have been peer-reviewed and accepted for publication. They are posted online prior to technical editing, formatting for publication and author proofing. The American Chemical Society provides "Just Accepted" as a service to the research community to expedite the dissemination of scientific material as soon as possible after acceptance. "Just Accepted" manuscripts appear in full in PDF format accompanied by an HTML abstract. "Just Accepted" manuscripts have been fully peer reviewed, but should not be considered the official version of record. They are citable by the Digital Object Identifier (DOI®). "Just Accepted" is an optional service offered to authors. Therefore, the "Just Accepted" Web site may not include all articles that will be published in the journal. After a manuscript is technically edited and formatted, it will be removed from the "Just Accepted" Web site and published as an ASAP article. Note that technical editing may introduce minor changes to the manuscript text and/or graphics which could affect content, and all legal disclaimers and ethical guidelines that apply to the journal pertain. ACS cannot be held responsible for errors or consequences arising from the use of information contained in these "Just Accepted" manuscripts. 


\title{
A Porous Covalent Organic Framework with Voided Square Grid Topology for Atmospheric Water Harvesting
}

\author{
Ha L. Nguyen, ${ }^{\dagger, \ddagger}$ Nikita Hanikel, ${ }^{\dagger, \ddagger}$ Steven J. Lyle, ${ }^{\dagger}$ Chenhui Zhu, ${ }^{\S}$ Davide M. Proserpio, ${ }^{\perp, \|}$ and Omar \\ M. Yaghi ${ }^{*},+, \nabla$ \\ †Department of Chemistry, University of California-Berkeley; Kavli Energy Nanoscience Institute at UC Berkeley; \\ Berkeley Global Science Institute; and Materials Sciences Division, Lawrence Berkeley National Laboratory, Berkeley, \\ California 94720, United States \\ §Advanced Light Source, Lawrence Berkeley National Laboratory, Berkeley, California 94720, United States \\ ${ }^{\perp}$ Dipartimento di Chimica, Università degli Studi di Milano, Milano 20133, Italy \\ "Samara Center for Theoretical Materials Science, Samara State Technical University, Samara 443100, Russia \\ ${ }^{\nabla}$ UC Berkeley-KACST Joint Center of Excellence for Nanomaterials for Clean Energy Applications, King Abdulaziz City \\ for Science and Technology, Riyadh 11442, Saudi Arabia
}

\section{Supporting Information Placeholder}

\begin{abstract}
Atmospheric moisture is a ubiquitous water resource available at any time and any place, making it attractive to develop materials for harvesting water from air to address the imminent water shortage crisis. In this context, we have been exploring the applicability of covalent organic frameworks (COFs) for water harvesting and report here a new porous, two-dimensional imine-linked COF with a voided square grid topology, termed COF-432. Unlike other reported COFs, COF-432 meets the requirements desired for water harvesting from air in that it exhibits an ' $S$ '-shaped water sorption isotherm with a steep pore-filling step at low relative humidity and without hysteretic behavior - properties essential for energy efficient uptake and release of water. Further, it can be regenerated at ultra-low temperatures and displays exceptional hydrolytic stability, as demonstrated by the retention of its working capacity after 300 water adsorption-desorption cycles.
\end{abstract}

Developing new materials for water harvesting from air is an important endeavor in addressing the global water crisis. ${ }^{1}$ Ideally, such materials should have: (i) high, hydrolytic stability and retention of capacity upon long-term water uptake and release cycling, (ii) an ' $S$ '-shaped water sorption isotherm with a steep pore-filling step (IUPAC Type IV or V) at low relative humidity $(<40 \%$ relative humidity, $\mathrm{RH})$ with minimal to no hysteresis, and (iii) a low regeneration temperature to allow for use of low-grade heating to enable facile release of water molecules from the material. ${ }^{2}$

Reticular structures, that is metal-organic frameworks (MOFs) and covalent organic frameworks (COFs), are ideally suited to address the water shortage crisis because of their exceptional porosity, as well as the large diversity of chemical compositions and accessible topologies; aspects that allow to tune their water sorption properties in a great variety of ways. ${ }^{2,3}$ Indeed, MOFs have been identified, studied, and practically employed for water harvesting from air.,4-9 COFs, however, remain largely unexplored for this application. ${ }^{10}$ This could potentially be explained by the relatively lower crystallinity exhibited by COFs, in particular those constructed from hydrolytically robust linkages, ${ }^{11}$ which precludes the formation of highly ordered molecular water networks within the porous framework - an important pre-requisite for obtaining ' $S$ '-shaped water isotherm profiles.

Herein, we sought to explore COFs as water harvesting materials. Accordingly, we report a new, highly crystalline framework, constructed from the tetratopic 1,1,2,2-tetrakis(4-aminophenyl)ethene [ETTA, $\left.\mathrm{C}_{26} \mathrm{H}_{16}\left(\mathrm{NH}_{2}\right)_{4}\right]$ and the tritopic 1,3,5-triformylbenzene $\left[\mathrm{TFB}, \mathrm{C}_{6} \mathrm{H}_{3}(\mathrm{CHO})_{3}\right]$, termed COF-432 $\left\{\left[(\mathrm{ETTA})_{3}\right.\right.$ (TFB) $\left.{ }_{4}\right]_{\text {imine }}$, which exhibits the mtf topology - a net hitherto not reported in COF chemistry (Figure 1). ${ }^{11-13}$ This COF displays a water sorption isotherm without hysteretic behavior and with a steep pore-filling step at low relative humidity $(<40 \% \mathrm{RH})$, exceptionally 
high water sorption cycling stability, and a low heat of adsorption. All these factors establish COF-432 as a long-term hydrolytically stable water harvesting material with a low regeneration energy barrier and relatively high working capacity within a small partial pressure range; with the latter enabling efficient use of a small temperature gradient for water uptake and release cycling.
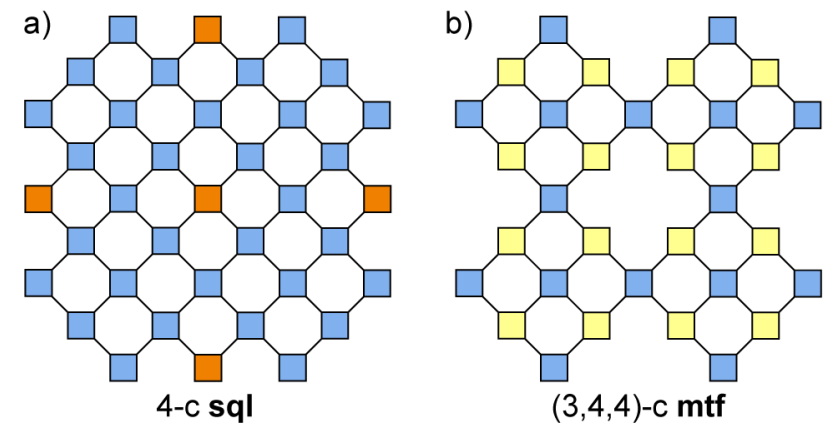

Figure 1. Juxtaposition of the 4-c uninodal sql net (a) and the $(3,4,4)$-c trinodal $\mathbf{m t f}$ net (b). The $\mathbf{m t f}$ net is conceptually constructed by removing $1 / 8$ of the nodes (orange) from the sql net, and can be perceived as a voided sql net. The nodes in both nets are depicted as squares. Blue squares represent 4-c and yellow squares 3-c nodes.

COF-432 was synthesized solvothermally through the condensation of ETTA and TFB in a mixture of chloroform, methanol, and aqueous acetic acid (Figure 2, Supporting Information, SI, Section S2). The structure of COF-432 was determined by powder X-ray diffraction (PXRD) and supported by elemental analysis (EA), Fourier-transform infrared (FTIR) spectroscopy, ${ }^{13} \mathrm{C}$ cross-polarization magic angle spinning nuclear magnetic resonance (CP-MAS NMR) spectroscopy, thermogravimetric analysis (TGA) and $\mathrm{N}_{2}$ sorption analysis. FTIR spectroscopy of COF-432 showed the absence of aldehyde $\left(v_{\mathrm{C}=0}=1692 \mathrm{~cm}^{-1}\right)$ and amine $\left(v_{\mathrm{N}-\mathrm{H}}=3352 \mathrm{~cm}^{-1}\right)$ stretches, present in the starting materials ETTA and TFB. Also, the emergence of an imine $\left(v_{\mathrm{C}=\mathrm{N}}=1628 \mathrm{~cm}^{-1}\right)$ stretch indicated the formation of an extended imine-linked network (SI, Section S3). Formation of the imine linkage was further corroborated by ${ }^{13} \mathrm{C}$ CP-MAS NMR spectroscopy, in which a characteristic ${ }^{13} \mathrm{C}$ imine resonance was observed at $158 \mathrm{ppm}$ (SI, Section S4).

Due to the small crystal size (ca. $100 \times 100 \times$ $300 \mathrm{~nm}^{3}$, SI, Section S5) of COF-432, its structure was determined by analysis of its PXRD pattern (SI, Section S6). Indexing of the PXRD pattern using TOPAS 4.2 software ${ }^{14}$ identified the space group $I 4_{1} / a$ (No. 88). Next, a Charge Flipping method ${ }^{15}$ was used to calculate the electron density map (EDM) of COF-432, generating a valid result in the respective space group. Finally, the structure of COF-432 was determined by locating the fragments of ETTA observed in the EDM (SI,
Section S6) and linking those into an extended network. In this structure, the ETTA and TFB building units (Figure 2a) are connected through imine bonds to form an extended two-dimensional (2D) framework of mtf topology (Figure 1, 2b, SI, Section S7). The unit cell parameters of COF-432 were refined by the Le Bail method using wide-angle X-ray scattering (WAXS) data $\left(I 4_{1} / a ; a=30.65 \AA\right.$, $\mathrm{c}=12.85 \AA$ A $)$ with residual factors of $R_{\mathrm{p}}=2.88 \%$ and $R_{\mathrm{wp}}=3.96 \%$ (Figure 3; SI, Section 6).

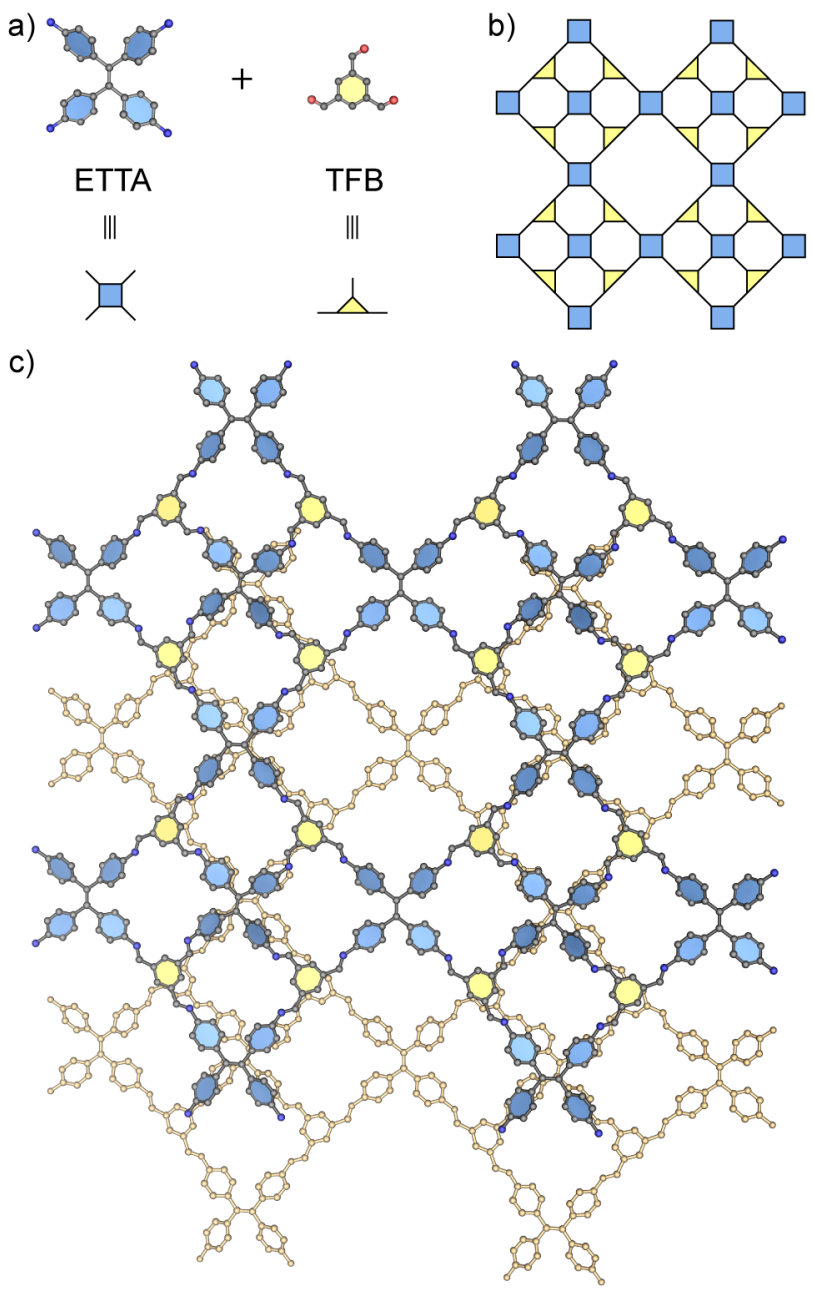

Figure 2. The reaction of 1,1,2,2-tetrakis(4-aminophenyl) ethene (ETTA) and 1,3,5-triformylbenzene (TFB) (a), which represent a 4-c and a 3-c node, respectively, yields COF-432 (c). This framework exhibits the $(3,4,4)$-c mtf topology, shown in its augmented form (b). Atom colors: $\mathrm{C}$, gray; $\mathrm{N}$, blue; $\mathrm{O}$, red. $\mathrm{H}$ atoms are omitted for clarity. The second layer of the staggered structure of COF432 is depicted in light orange.

A single layer of COF-432 (Figure 2c) has two kind of square pore apertures with diameters of ca. $10.0 \AA$ and $21.0 \AA$ (based on van der Waals radii). Adjacent 2D layers are staggered, thus creating a 1D cylindrical pore structure with a diameter of ca. $7.5 \AA$ (Figure 2c). COF-432 is permanently porous with a BET surface area of $895 \mathrm{~m}^{2} \mathrm{~g}^{-1}$. This is close to the theoretical value 
calculated from the structural model $\left(900 \mathrm{~m}^{2} \mathrm{~g}^{-1}\right)$, approximated by its molecularly accessible area using $\mathrm{N}_{2}$ as the probe adsorbate (kinetic diameter $=3.6 \AA$ ) ${ }^{16}$ The pore volume of COF-432, determined from its $\mathrm{N}_{2}$ sorption isotherm $\left(0.43 \mathrm{~cm}^{3} \mathrm{~g}^{-1}\right)$, is in good agreement with the pore volume predicted from its structural model using the Void Calculation function in PLATON $\left(0.45 \mathrm{~cm}^{3} \mathrm{~g}^{-1}\right)$. Additionally, the pore size distribution of COF-432 calculated from its $\mathrm{N}_{2}$ sorption isotherm indicates a single pore with a diameter of $8.0 \AA$, which is in good agreement with the proposed crystal structure (SI, Section S8). The structural model is further confirmed by elemental analysis of COF-432, which matches well with the expected elemental ratio calculated for the framework (SI, Section S2).

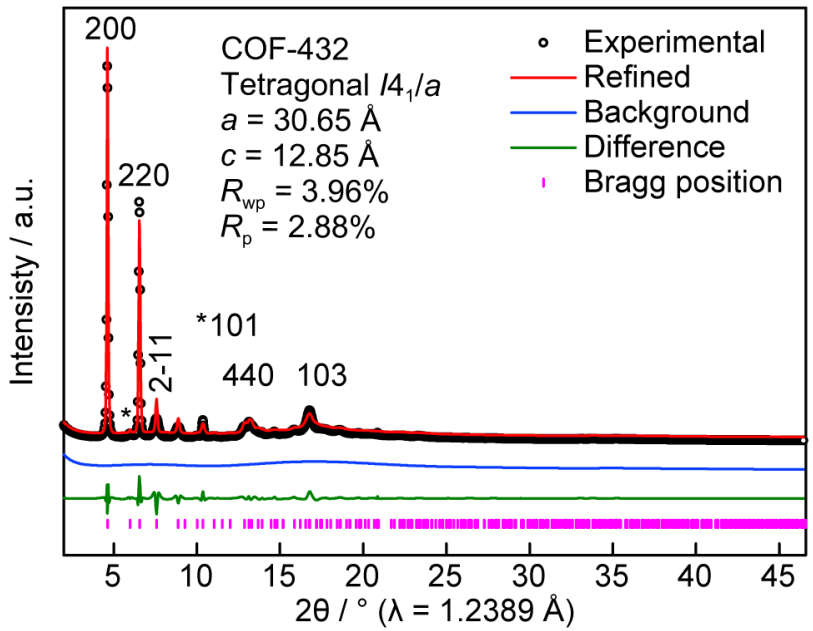

Figure 3. Wide-angle X-ray scattering (WAXS) pattern and Le Bail analysis of COF-432. The experimental pattern (black), the refined Le Bail fitting (red), the difference plot (green), background (blue), and the Bragg positions (pink) are provided.

The hydrolytic stability of COF-432 was initially investigated by immersing the activated $\mathrm{COF}$ in water. PXRD patterns of the framework before and after exposure demonstrated that the material retained its crystallinity for at least $20 \mathrm{~d}$. Additionally, the material did not lose its surface area after extended soaking of the COF in water ( $90 \mathrm{~h}$ under stirring; SI, Section S10). We hypothesize that this stability stems from tight packing of the 2D COF layers and usage of high-connectivity linker molecules, which hampers the hydrolysis of the labile imine bonds.

The exceptional hydrolysis resistance of COF- 432 encouraged us to study its water sorption properties. The framework exhibits an ' $S$ '-shaped water sorption isotherm with a steep pore-filling step at 34\% RH (at $25^{\circ} \mathrm{C}$; Figure 4a). The maximal water uptake at $P / P_{\text {sat }}$ $=0.95$ reaches $30 \mathrm{wt} \%\left(0.3 \mathrm{~g} \mathrm{~g}^{-1}\right)$ and the working capacity in the relative humidity range between 20 and $40 \%$ is $0.23 \mathrm{~g} \mathrm{~g}^{-1}$. Unlike other COFs, COF- 432 does not exhibit hysteretic water sorption behavior (Figure $4 \mathrm{a}$,
SI, Section S11). This is an attractive feature because it restricts the energy requirement for regeneration of this material. To further study the interaction of the water molecules with the $\mathrm{COF}$, water sorption isotherms at different temperatures $\left(10,25\right.$, and $40{ }^{\circ} \mathrm{C}$; Figure 4a) were used to calculate the isosteric heat of adsorption of water in COF-432. It was estimated to account to ca. $48 \mathrm{~kJ} \mathrm{~mol}^{-1}$ (SI, Section S11) - close to the evaporation enthalpy of water $\left(44 \mathrm{~kJ} \mathrm{~mol}^{-1}\right.$ at $25^{\circ} \mathrm{C}$ ). This indicates that water-water interactions are predominant during the pore filling process. ${ }^{2}$ Indeed, the pore surface of COF-432 is mostly non-polar, however, the imine bonds could serve as primary adsorption sites for water molecules, as weak hydrogen bonding between imine-bonds of a COF and water molecules has been observed in a previous study. ${ }^{17}$ This weak hydrogen bonding and the strong confinement effect in the small framework pores (ca. $7.5 \AA$ diameter) likely initiate the pore-filling step at low relative humidity $(<40 \% \mathrm{RH})$. We hypothesize that the absence of highly polar functional groups and low framework flexibility through employment of high-
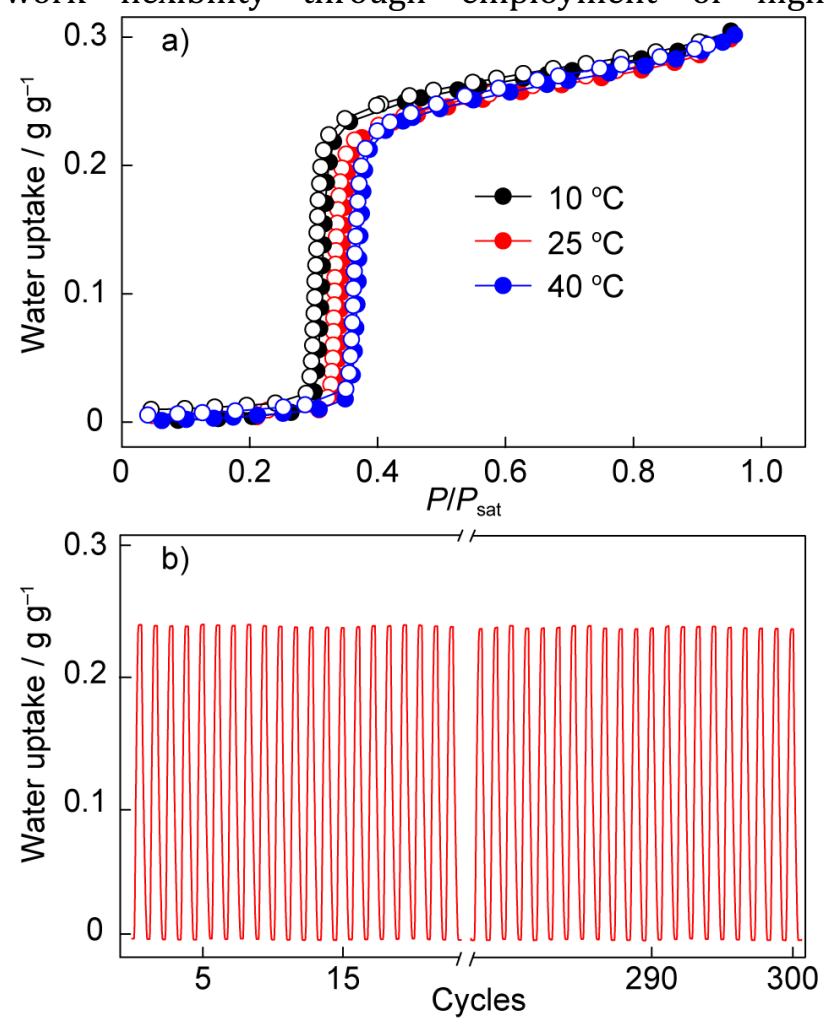

Figure 4. (a) Water sorption analysis on COF-432, measured at different temperatures $\left(10,25\right.$ and $\left.40{ }^{\circ} \mathrm{C}\right)$. $P$ : water vapor pressure. $P_{\text {sat: }}$ saturation water vapor pressure at the given temperature. (b) Water cycling stability test for 300 adsorption-desorption cycles conducted on COF432 at constant water vapor pressure $(1.7 \mathrm{kPa})$. Adsorption and desorption are carried out at $30{ }^{\circ} \mathrm{C}(40 \%$ relative humidity, $\mathrm{RH})$ and $35^{\circ} \mathrm{C}(30 \% \mathrm{RH})$, respectively (full data set is displayed in the SI, Section S12). 
connectivity (tri- and tetratopic) linker molecules prevent hysteretic water sorption behavior of COF432. 18,19

Importantly, COF-432 retained its crystallinity, BET surface area and water vapor capacity after 7 consecutive water sorption measurements (SI, Section S12) - an impressive feat considering that other COFs reported as promising water sorbents in the literature exhibit a decrease in surface area after water exposure and/or sorption. ${ }^{20-23}$ These findings encouraged us to subject COF-432 to a long-term water adsorption-desorption cycling test: In a thermogravimetric analyzer, the framework was exposed to water vapor under isobaric conditions (1.7 $\mathrm{kPa})$, and a temperature swing between 30 and $35{ }^{\circ} \mathrm{C}$ (corresponding to 40 and 30\% $\mathrm{RH}$, respectively) was applied to trigger water ad-and desorption. The steep pore-filling step allowed for a high working capacity of $0.23 \mathrm{~g} \mathrm{~g}^{-1}$ under employment of an ultra-low temperature gradient $\left(5^{\circ} \mathrm{C}\right)$. In total, 300 uptake and release cycles were conducted, and the working capacity remained unchanged during the experiment (Figure 4b), indicating retention of porosity and thus exceptional water cycling stability.

In summary, we have synthesized a new 2D iminelinked framework, COF-432, which exhibits attractive water sorption properties. In particular, COF-432 has (i) exceptional long-term stability upon water uptake and release cycling, (ii) a hysteresis-free water sorption isotherm with a steep uptake step at low relative humidity, and (iii) low heat of adsorption, allowing for regeneration by low-grade energy sources. Accordingly, COF-432 could be employed as material for water harvesting from air, and potentially in heat pump systems or in desiccant-based dehumidifiers.

We note that selected reported MOFs exhibit a higher water uptake capacity than COF-432, while the other characteristics (hydrolytic stability, isotherm profile, facile regeneration) are comparable with the most advanced water harvesting $\mathrm{MOFs}^{2}{ }^{2}$ indicating that COFs can, indeed, be competitive materials in this regard. Additionally, the usage of all-organic materials does not rely on metal cations, which may potentially exhibit toxic properties or be cost-prohibitive. In conclusion, we think that extending the scope of material classes suitable for atmospheric water extraction will be of great benefit to this technology, and we firmly believe that our findings will inspire more future research on COFs as water harvesting materials.

\section{ASSOCIATED CONTENT}

\section{Supporting Information.}

The Supporting Information is available free of charge on the ACS Publications website.

Synthesis and characterization details of COF432: Powder X-ray diffraction analysis data, computational modeling, and water sorption analysis data (PDF)

\section{AUTHOR INFORMATION}

\section{Corresponding Author}

O.M.Y.: yaghi@berkeley.edu.

\section{Author Contributions}

${ }^{*}$ H.L.N. and N.H. contributed equally.

\section{Notes}

O.M.Y. is co-founder of Water Harvesting Inc., aiming at commercializing related technologies.

\section{ACKNOWLEDGMENT}

We acknowledge King Abdulaziz City for Science and Technology as part of a joint KACST-UC Berkeley collaboration. N.H. would like to thank the Studienstiftung des deutschen Volkes. D.M.P. thanks the Università degli Studi di Milano for the transition grant PSR2015-1718 and FFABR2018. This research used beamline 7.3.3 of the Advanced Light Source, which is a DOE Office of Science User Facility under contract no. DE-AC0205CH11231. We thank Mr. Hao Lyu (Yaghi group) for the SEM measurement and useful discussions. Additionally, we would like to thank Prof. Valentina Colombo (Università degli Studi di Milano) and Dr. Cornelius Gropp (Yaghi group) for useful discussions. We acknowledge Prof. Xiaodong Zou and Dr. Zhehao Huang (Stockholm University) for preliminary efforts in structural elucidation.

\section{REFERENCES}

(1) Wahlgren, R. V. Atmospheric water vapour processor designs for potable water production: A review. Water Res. 2001, 35, 1-22.

(2) Kalmutzki, M. J.; Diercks, C. S.; Yaghi, O. M. Metal-organic frameworks for water harvesting from air. Adv. Mater. 2018, 30, 1704304 .

(3) Yaghi, O. M.; Kalmutzki, M. J.; Diercks, C. S. Introduction to Reticular Chemistry: Metal-organic frameworks and covalent organic frameworks, Wiley-VCH, Weinheim, 2019, 509.

(4) Burtch, N. C.; Jasuja, H.; Walton, K. S. Water stability and adsorption in metal-organic frameworks. Chem. Rev. 2014, 114, 10575-10612.

(5) Rieth, A. J.; Yang, S.; Wang, E. N.; Dincă, M. Record atmospheric fresh water capture and heat transfer with a material operating at the water uptake reversibility limit. ACS Cent. Sci. 2017, 3, 668-672.

(6) Kim, H.; Yang, S.; Rao, S. R.; Narayanan, S.; Kapustin, E. A.; Furukawa, H.; Umans, A. S.; Yaghi, O. M.; Wang, E. N. Water harvesting from air with metal-organic frameworks powered by natural sunlight. Science, 2017, 356, 430-434.

(7) Kim, H.; Rao, S. R.; Kapustin, E. A.; Zhao, L.; Yang, S.; Yaghi, O. M.; Wang, E. N. Adsorption-based atmospheric water harvesting device for arid climates. Nat. Commun. 2018, 9, 1191.

(8) Fathieh, F.; Kalmutzki, M. J.; Kapustin, E. A.; Waller, P. J.; Yang, J.; Yaghi, O. M. Practical water production from desert Air. Sci. Adv., 2018, 4, eaat3198.

(9) Hanikel, N.; Prévot, M. S.; Fathieh, F.; Kapustin, E. A.; Lyu, H.; Wang, H.; Diercks, N. J.; Glover, T. G.; Yaghi, O. M. Rapid cycling and exceptional yield in a metal-organic frame-work water harvester. ACS Cent. Sci. 2019, 5, 1699-1706. 
(10) Byun, Y.; Je, S. H.; Talapaneni, S. N.; Coskun, A. Advances in porous organic polymers for efficient water capture. Chem. Eur. J. 2019, 25, 10262-10283.

(11) Lohse, M. S.; Bein, T. Covalent organic frameworks: Structures, synthesis, and applications. Adv. Funct. Mater. 2018, 28,1705553

(12) Lan, Y.; Han, X.; Tong, M.; Huang, H.; Yang, Q.; Liu, D.; Zhao, X.; Zhong, C. Materials genomics methods for high-throughput construction of COFs and targeted synthesis. Nat. Commun. 2018, 9, 5274.

(13) Lyle, S. J.; Waller, P. J.; Yaghi, O. M. Covalent organic frameworks: Organic chemistry extended into two and three dimensions. Trends Chem. 2019, 1, 172-184.

(14) Bruker AXS GmbH, TOPAS Manual: DOC-M88-EXX065 V4.2 $-01.2009$.

(15) Palatinus, L.; Chapuis, G. SUPERFLIP-A Computer program for the solution of crystal structures by charge flipping in arbitrary dimensions. J. Appl. Crystallogr. 2007, 40, 786790.

(16) Düren, T.; Millange, F.; Férey, G.; Walton, K. S.; Snurr, R. Q. Calculating geometric surface areas as a characterization tool for metal-organic frameworks. J. Phys. Chem. C 2007, 111, 15350-15356.

(17) Ma, T.; Kapustin, E. A.; Yin, S. X.; Liang, L.; Zhou, Z.; Niu, J.; Li, L. H.; Wang, Y.; Su, J.; Li, J.; Wang, X.; Wang, X. D.; Wang, W.; Sun, J.; Yaghi, O. M. Single-crystal X-ray diffraction structures of covalent organic frameworks. Science 2018, 361, 48-52.
(18) Furukawa, H.; Gándara, F.; Zhang, Y. B.; Jiang, J.; Queen, W. L.; Hudson, M. R.; Yaghi, O. M. Water adsorption in porous metal-organic frameworks and related materials. J. Am. Chem. Soc. 2014, 136, 4369-4381.

(19) Canivet, J.; Bonnefoy, J.; Daniel, C.; Legrand, A.; Coasne, B.; Farrusseng, D. Structure-property relationships of water adsorption in metal-organic frameworks. New J. Chem. 2014, 38, 3102-3111.

(20) Biswal, B. P.; Kandambeth, S.; Chandra, S.; Shinde, D. B.; Bera, S.; Karak, S.; Garai, B.; Kharul, U. K.; Banerjee, R. Pore surface engineering in porous, chemically stable covalent organic frameworks for water adsorption. J. Mater. Chem. A 2015, 3, 23664-23669.

(21) Stegbauer, L.; Hahn, M. W.; Jentys, A.; Savasci, G.; Ochsenfeld, C.; Lercher, J. A.; Lotsch, B. V.; Tunable water and $\mathrm{CO}_{2}$ absorption properties in isostructural azine-based covalent organic frameworks through polarity engineering. Chem. Mater. 2015, 27, 7874-7881.

(22) Karak, S.; Kandambeth, S.; Biswal, B. P.; Sasmal, H. S.; Kumar, S.; Pachfule, P.; Banerjee, R. Constructing ultraporous covalent organic frameworks in seconds via an organic terracotta process. J. Am. Chem. Soc. 2017, 139, 1856-1862.

(23) Pérez-carvajal, J.; Boix, G.; Imaz, I.; Maspoch, D. The iminebased COF TpPa-1 as an efficient cooling adsorbent that can be regenerated by heat or light. Adv. Energy Mater. 2019, 1901535 


\section{Table of Contents}

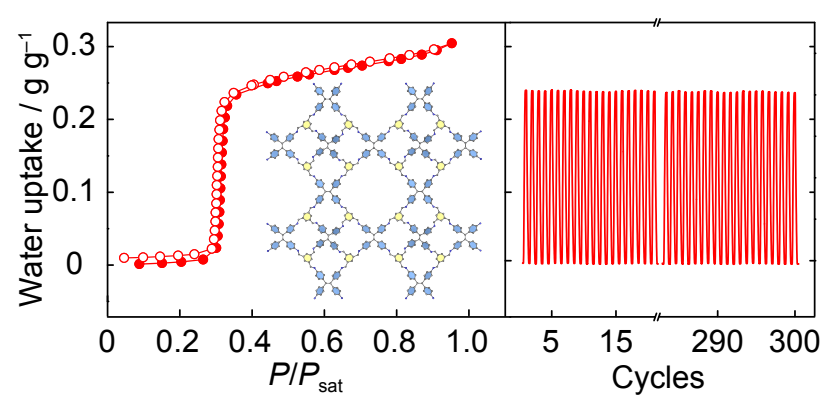

